\title{
Rectal Injury by Inserting a Vaginal Pipe for Laparoscopic Hysterectomy
}

\author{
Keiko Mekaru*, Sugiko Oishi, Kozue Akamine, Chiaki Heshiki, and Yoichi Aoki \\ Department of Obstetrics and Gynecology, Graduate School of Medical Science, University of the Ryukyus, 207 \\ Uehara, Nishihara, Okinawa 903-0215, Japan
}

*Corresponding Author: Keiko Mekaru, Department of Obstetrics and Gynecology, Graduate School of Medical Science, University of the Ryukyus, 207 Uehara, Nishihara, Okinawa 903-0215, Japan, Email: f041252@eve.u-ryukyu.ac.jp

\begin{abstract}
Introduction: We describe a preventable rectal injury from unexpected error in total laparoscopic hysterectomy. We should pay attention to insert the vaginal pipe to vagina accurately.

Case Report: We present a 38-year-old woman (1 gravida, 1 para) with hypermenorrhea that resulted in anemia. We performed a total laparoscopic hysterectomy because of a 3-cm uterine submucosal myoma. There were no adhesions in the upper abdomen and pelvis. We planned to insert a vaginal pipe to identify the border of the uterine fornix and vagina; however, it was accidentally inserted into the rectum, and the incision was made before identifying the rectum. After the 3-cm rectal incision, we recognized the mucosa of the rectum. A lower anterior resection of the rectum with laparotomy was performed. The patient abstained from food and was on intravenous hyperalimentation and was discharged 2 weeks after surgery.
\end{abstract}

Discussion: Inserting a vaginal pipe to rectum is unexpected error; however, it will cause severe complication. We should examine the location of the vagina and insert the pipe correctly into the vagina with proper lighting.

Keywords: Laparoscopic surgery, rectal injury, hysterectomy, vaginal pipe, gynecological surgery

\section{INTRODUCTION}

Laparoscopic hysterectomy (LH) is preferred for benign diseases when it is not possible to perform vaginal hysterectomy [1]. In comparison with abdominal hysterectomy, the time to resume normal activities after LH was shorter, but urinary tract injuries were more frequent after LH [1].Patients prefer to undergo procedures involving less pain, smaller scars, and faster recovery, which are the benefits of laparoscopic surgery. However, even small errors may lead to severe complications in laparoscopic surgeries. We herein describe a preventable rectal injury caused during total LH (TLH). Insertion of a vaginal pipe in the rectum is an unusual occurrence and the authors' have never previously encountered a similar case. Knowing that a vaginal pipe may insert rectally with ease if enough caution is not taken is essential to prevent this error. For this reason, we decided to present the current case. This study was conducted according to the guidelines of the Declaration of Helsinki and was approved by the Institutional Review Board of the University of Ryukyu. The patient provided informed consent.

\section{CASE REPORT}

A 38-year-old woman (gravida 1, para 1) had hypermenorrhea that resulted in anemia. Since the patient had a narrow vaginal canal, we performed TLH for a 3-cm uterine submucosal myoma detected via ultrasonography and magnetic resonance imaging. After general anesthesia was induced, the patient was placed in a low lithotomy position. The camera port of a trocar was inserted into the umbilicus; three ports were inserted into the lower abdomen. The uterus was $11 \mathrm{~cm} \times 8 \mathrm{~cm}$ in size, and uterine mobility was good. An intrauterine manipulating device was inserted so that the uterus can be manipulated during the laparoscopy. Bilateral round ligaments, tubes, and utero-ovarian ligaments were localized, ligated and cut using bipolar forceps and an electrical scalpel. After determining the location 
of the uterine vessels, the ureters were confirmed to be under uterine artery. Bilateral uterine arteries were ligated and cut. Next, the bladder was dissected from the uterine cervix. Finally, the vaginal pipe (Hakko Co., Ltd. Nagano, Japan) was inserted as a delineator for identifying the border between the uterine fornix and the vagina for colpotomy (Figure 1).

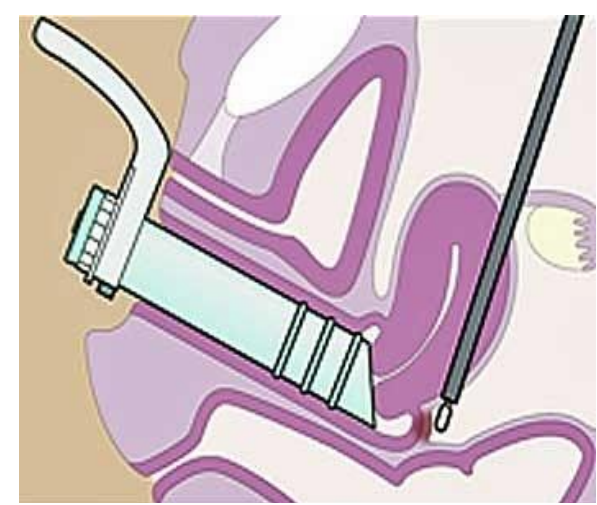

Figure1. Vaginal pipe inserted correctly in the vagina.

However, in this case, the vaginal pipe was inserted into the rectum, and an incision was made without rectal identification (Figure 2).
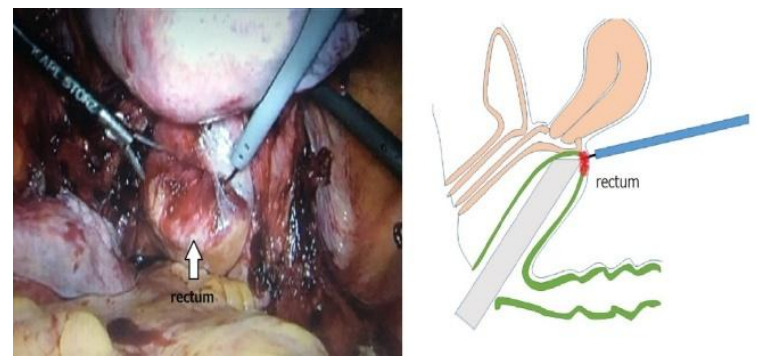

Figure2. (a). Vaginal pipe inserted into the rectum

(b).Illustration of the vaginal pipe inserted into the rectum

The rectal mucosa was identified only after a 3$\mathrm{cm}$ rectal incision was made (Figure3).

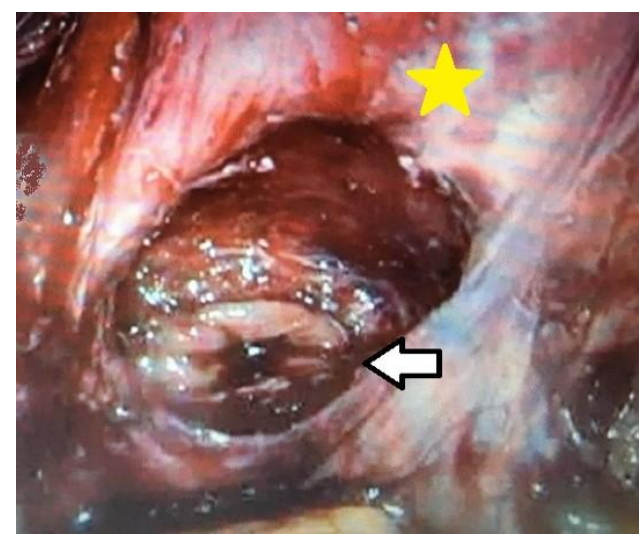

Figure3. Observation of rectal injury. $\diamond:$ Findings of rectal injury, $\star$. fornix of the uterine cervix

A lower anterior rectal resection with laparotomy was performed. The patient was placed on intravenous hyperalimentation and discharged 2 weeks after the surgery.

\section{DISCUSSION}

Gynecologic laparoscopy is being increasingly performed. In Japan, this procedure has been performed in 1,$482 ; 9,946$; and 44,483 cases in 1990, 2000, and 2013, respectively [2].Conversely, TLH was performed in 12,857 cases between 2012 and 2013; there were bowel injuries in 24 cases [2].

There were bowel injuries in 604 cases among 474,063 laparoscopic surgeries performed between 1972 and 2014 [3].Among the 354 bowel injuries reported in the gastrointestinal tract, the most frequently damaged region was the small intestine (47\%) followed by the colon $(30 \%)$, rectum (18\%), and stomach (6\%) [3].During laparoscopic surgery, instruments have an important role in the success of the procedure, and various devices are available for this purpose $[4,5] . H o w e v e r$, some instruments may cause damage to organs, such as vessels, bladder, and bowel. Of the 366 bowel injuries described where laparoscopic instruments were responsible for the damage, the majority of bowel injuries occurred during abdominal access and insufflation using a Veress needle or during trocar placement (55\%). Injuries associated with electrosurgery occurred in $29 \%$ of the cases [3].

There are two reports on bowel injuries associated with vaginal pipes. Asai et al[3].reported rectal injury in a patient with correct vaginal pipe insertion, where the vaginal delineator was placed posteriorly because the vagina lacked distensibility, which led to a false presentation of the vagina and rectum. Okamoto et al. [7] reported a case similar to ours. They inserted a vaginal pipe into the rectum and injured the rectum. Laparotomy was not performed because the rectal perforation was small, and the injury could be repaired laparoscopically.

In our case, rectal injury occurred because a simple examination was not performed. However, the cause based on the individual matrices of the surgeon, patient, instrument, and setting should be considered to prevent similar incidents. The surgeon who inserted the vaginal pipe had 10 years of experience as an obstetrician. However, she had little experience with laparoscopic surgery; this was the third time she was performing vaginal pipe insertion. The patient's vagina and anus were in the 
correct position, and there was no stenosis in the vagina. The rectum is generally narrow and firmer than the vagina; thus, vaginal pipe insertion into the rectum is more difficult; however, under general anesthesia, the rectum was flaccid enough to insert the pipe without resistance. With regard to the setting, there was no lighting to the vagina when the vaginal pipe was inserted into the rectum; proper lighting is necessary during pipe insertion.

\section{CONCLUSION}

In conclusion, inserting a vaginal pipe in the rectum may lead to severe complications. Knowing that a vaginal pipe may insert rectally with ease if enough caution is not taken is essential to prevent this error.

\section{ACKNOWLEDGMENT}

The authors would like to thank Enago (https://www.enago.jp) for the English language review

\section{REFERENCES}

[1] Aarts J.W., Nieboer T.E., Johnson N., et al. Surgical approach to hysterectomy for benign gynecological disease, Cochrane Database Syst Rev. 2015; 8(3): CD003677. DOI 10.1002/ 14651858.CD003677.pub4.
[2] Kitano M.,Survey on endoscopic surgery, JSES. 2014;Sep:19(5):592-598

[3] Llarena N.C., Shah A.B., and Milad M.P.,Bowelinjury in gynecologic laparoscopy: a systematic review, Obstet Gynecol. 2015; 125(5):1407-1417. DOI10.1097/ AOG.000000 0000000855 .

[4] Ramirez P.T., Frumovitz M., Dos Reis R., et al. Modified uterine manipulator and vaginal rings for total laparoscopic radical hysterectomy, Int J Gynecol Cancer. 2008;18(3):571-5.DOI: 10.1111/j.1525-1438.2007.01038.x.

[5] Tanprasertkul C. and Kulvanitchaiyanunt A., A modified technique to simplify TLH with new developed uterine manipulator; Anurach uterine manipulator (AUM), J Med Assoc Thai. 2010;; 93(7):S154-S159.

[6] Asai S., Ishimoto H., Okuno S., et al. Rectal injury associated with insertion of a

[7] vaginal delineator tube during total laparoscopic hysterectomy: A case report and review of the literature, GMIT. 2014; 3: 54-56.

[8] Okamoto S., Nakanishi K., Ono M., et al.Rectal perforation after incision of the

[9] vaginal canal following incorrect insertion of a Vagi-Pipe into the rectum during total laparoscopic hysterectomy, J Onstet Gynecol. 2016; 36:992-993.

Citation: Keiko Mekaru, Sugiko Oishi, Kozue Akamine, Chiaki Heshiki, Yoichi Aoki. Rectal Injury by Inserting a Vaginal Pipe for Laparoscopic Hysterectomy. ARC Journal of Gynecology and Obstetrics 2017; 2(2):15-17. DOI: dx.doi.org/10.20431/2455-9792.0202004

Copyright: () 2017 Authors. This is an open-access article distributed under the terms of the Creative Commons Attribution License, which permits unrestricted use, distribution, and reproduction in any medium, provided the original author and source are credited. 